\title{
Negative refraction and energy funneling by hyperbolic materials: An experimental demonstration in acoustics
}

\author{
García-Chocano, Victor M.; Christensen, Johan; Sánchez-Dehesa, José
}

Published in:

Physical Review Letters

Link to article, DOI:

10.1103/PhysRevLett.112.144301

Publication date:

2014

Document Version

Publisher's PDF, also known as Version of record

Link back to DTU Orbit

Citation (APA):

García-Chocano, V. M., Christensen, J., \& Sánchez-Dehesa, J. (2014). Negative refraction and energy funneling by hyperbolic materials: An experimental demonstration in acoustics. Physical Review Letters, 112(14),

[144301]. https://doi.org/10.1103/PhysRevLett.112.144301

\section{General rights}

Copyright and moral rights for the publications made accessible in the public portal are retained by the authors and/or other copyright owners and it is a condition of accessing publications that users recognise and abide by the legal requirements associated with these rights.

- Users may download and print one copy of any publication from the public portal for the purpose of private study or research.

- You may not further distribute the material or use it for any profit-making activity or commercial gain

- You may freely distribute the URL identifying the publication in the public portal 


\title{
Negative Refraction and Energy Funneling by Hyperbolic Materials: An Experimental Demonstration in Acoustics
}

\author{
Victor M. García-Chocano, ${ }^{1}$ Johan Christensen, ${ }^{2}$ and José Sánchez-Dehesa ${ }^{1, *}$ \\ ${ }^{1}$ Wave Phenomena Group, Department of Electronics Engineering, Universitat Politècnica de València, \\ Camino de Vera s.n. (Edificio 7F), ES-46022 Valencia, Spain \\ ${ }^{2}$ Department of Photonics Engineering, Technical University of Denmark, \\ Orsteds Plads, Building 343, DK-2800 Kongens Lyngby, Denmark
}

(Received 17 January 2014; published 10 April 2014)

\begin{abstract}
This Letter reports the design, fabrication, and experimental characterization of hyperbolic materials showing negative refraction and energy funneling of airborne sound. Negative refraction is demonstrated using a stack of five holey Plexiglas plates where their thicknesses, layer separation, hole diameters, and lattice periodicity have been determined to show hyperbolic dispersion around $40 \mathrm{kHz}$. The resulting hyperbolic material shows a flat band profile in the equifrequency contour allowing the gathering of acoustic energy in a broad range of incident angles and its funneling through the material. Our demonstrations foresee interesting developments based on both phenomena. Acoustic imaging with subwavelength resolution and spot-size converters that harvest and squeeze sound waves irradiating from many directions into a collimated beam are just two possible applications among many.
\end{abstract}

A hyperbolic material has a characteristic feature: the wave vector of a propagating plane wave follows the surface of a hyperboloid in contrast to an ellipsoid, which is the surface associated with the propagation inside a conventional anisotropic material. In optics, a variety of interesting applications have been already described using hyperbolic materials $[1,2]$.

In their acoustic counterpart, artificial structures with hyperbolic dispersion have been already employed to measure subwavelength resolution [3]. Hyperbolic dispersion for airborne sound can be obtained in two dimensions (2D) using acoustic metamaterials based on anisotropic lattices of cylindrical scatterers embedded in a fluid [4]. Hyperbolic metamaterials showing negative acoustic refractive index can be obtained with properly designed scattering units [4].

A recent proposal $[5,6]$ uses periodically perforated hard plates to obtain hyperbolic dispersion of sound waves. Negative refraction is theoretically obtained at certain incident angles tilted with respect to normal incidence. Energy funneling is also another phenomenon associated to these extremely simple structures if the hyperbolic dispersion shows a flat profile. In spite of the numerous interesting applications foreseen for these materials in acoustics, scarce experimental works have been reported for airborne sound using those simple structures.

This Letter reports measurements showing negative refraction and energy funneling in artificial structures consisting of perforated Plexiglas plates embedded in air. Our demonstrations comprise not only new material properties with striking wave phenomena but also applications that could open the road of developing actual devices based on this simple structure.
The design of the holey structure leading to negative refraction was performed following the model introduced in Refs. [5,6]. In brief, the model considers an infinite stack of rigid plates like the Plexiglas plates described in Fig. 1. Analytical expressions giving the acoustic band structure of the hyperbolic material are developed using a few simplifying assumptions [6]. First, the structure is perfectly rigid and, therefore, the sound waves only propagate in air and the normal derivative of acoustic pressure vanishes at the plate and hole-wall surfaces. This assumption is amply fulfilled for Plexiglas due to the huge mismatch of its acoustic impedance with that of air. Second, we also consider that the holes diameter is much smaller than the air wavelength, implying that only monopolar modes are excited at each hole. Finally, the distances between nearest-neighbor holes in the plate and between consecutive plates are assumed large enough to consider that the response of each hole is given by the amplitudes of the scattering monopoles that it produces on its upper and lower parts. The reader is referred to Ref. [6], where details of the expressions obtained using these simplifications are given.

The analytical model was employed to determine the geometrical parameters of the structure $\left(D, a_{x}, a_{z}, t, s\right)$ giving rise to a hyperbolic dispersion at around $40 \mathrm{kHz}$. The inset in Fig. 2 shows the equifrequency surfaces (EFS) calculated at $41 \mathrm{kHz}$ for pressure waves propagating in free space (circular blue curve) and inside the infinite hyperbolic material (red continuous lines). The dotted lines plotted near the continuous ones represent EFS with slightly larger frequency. They are added in order to illustrate the directions (short arrows) of the group velocity $\mathbf{v}_{g}=\nabla_{\mathbf{k}} \omega$, which indicates that the energy flow is normal 

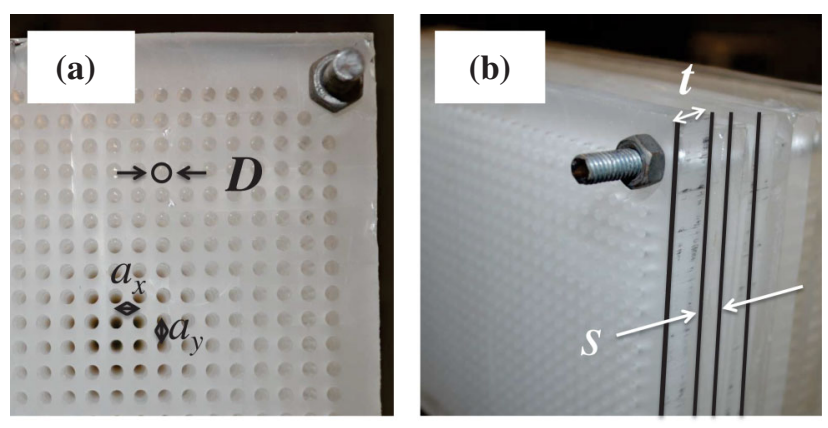

(c)

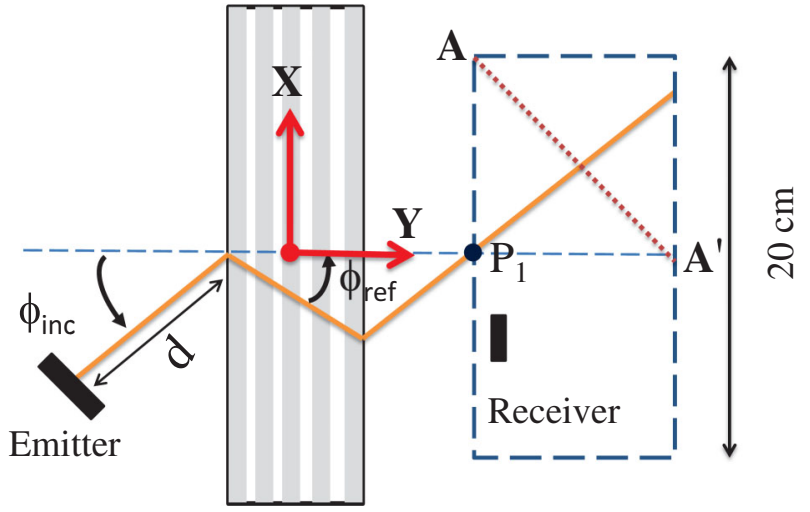

FIG. 1 (color online). (a) Frontal and (b) lateral views of the fabricated sample. It consists of a stack of holey Plexiglas plates with thickness $t=7.27 \mathrm{~mm}$ and separation $s=3.18 \mathrm{~mm}$. The holes of diameter $D=2.7 \mathrm{~mm}$ are drilled in a square distribution with lattice period $a_{x}=a_{y}=4.54 \mathrm{~mm}$. The hole periodicity along the normal is $a_{z}=t+s=10.45 \mathrm{~mm}$. (c) Scheme (not to scale) of the experimental setup. The rectangle defined by the dashed lines defines the area scanned by the receiver-transducer. The sample represented consists of five holey plates (gray rectangles) with total thickness $L$.

to the EFS in wave-vector space. From the EFS it is easy to predict the transmission behavior at the air-material interface. Thus, pressure waves propagating in free space with an angle $\phi_{\text {inc }}$ tilted with respect to the $z$ axis (thin blue arrow) will conserve the parallel component of the wave vector across the interface. For the hyperbolic material, the angle conserving $k_{\|}$(thin red arrow) will propagate inside the structure following the direction represented by the thick red arrow, which corresponds to a negative value of $\phi_{\text {ref }}$ indicating negative refraction. It is also concluded from the EFS that negative refraction is predicted for a broad range of incident angles (see Fig. 2).

In addition, the hyperbolic EFS shows a broad flat profile indicating that for a wide range of incident wave vectors the resulting propagating direction inside the material is perpendicular to its surface, i.e., $\phi_{\text {ref }}=0^{\circ}$. This feature means that the designed material is capable of collecting waves arriving to the material surface from many different directions, collimating them along the normal and funnel the energy through the bulk material.

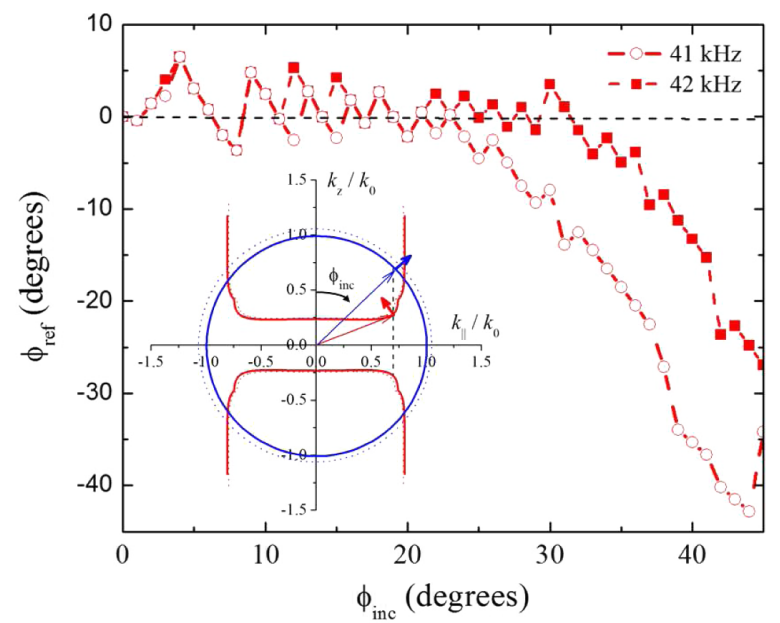

FIG. 2 (color online). Calculated propagating angle $\phi_{\text {ref }}$ inside the hyperbolic material described in Fig. 1 as a function of the incident angle $\phi_{\text {ref }}$. The inset shows the equifrequency curves corresponding to airborne sound propagating in free space (circle of radius $\left.k_{0}=2 \pi / \lambda_{0}\right)$ and inside a hyperbolic material with an infinite number of layers. $k_{\|}$and $k_{z}$ are the components of the wave-vector parallel and perpendicular, respectively, to the stack of Plexiglas layers. In terms of wavelength, $\lambda / D=3.2$, $\lambda / d_{x}=\lambda / d_{y}=1.9$, and $\lambda / d_{z}=0.8$. The vertical dashed line is a guide for the eye indicating the conservation of $k_{\|}$.

The two phenomena described above are supported by numerical simulations using the finite-element-method program COMSOL MuLTIPHYSICS. The calculations were performed using the configuration schematically depicted in Fig. 1(c) where the impinging sound was simulated with a Gaussian beam having the same thickness than the emitter transducer. Further details of COMSOL simulations are found in the Supplemental Material [7]. Simulations were conducted for many different incident angles, $\phi_{\text {inc }}$. The calculated refracted angles $\phi_{\text {ref }}$ as a function of the incident angles $\phi_{\text {inc }}$ are represented in Fig. 2 for two different frequencies. These results support the predictions embedded in the EFS described in the inset, which indicates the existence of a broad range of impinging angles for which the propagation is perpendicular to the surface $\phi_{\text {ref }}=0$, and another broad range of incident directions in which negative refraction is obtained $\phi_{\text {ref }}<0$.

Figure 3(a) presents a typical simulation showing the snapshot of the pressure map calculated for an impinging beam with frequency $41 \mathrm{kHz}$ and tilted $45^{\circ}$ with respect to the normal. Note that the position of the outgoing beam clearly indicates a negative angle of refraction inside the sample. From this data $\phi_{\text {ref }}$ can be extracted, corresponding to a specific point in the curves shown in Fig. 2. A motion picture depicting the incident and scattered pressure fields (with frequency fixed at $41 \mathrm{kHz}$ ) as a function of $\phi_{\text {inc }}$ is presented in the Supplemental Material Movie S1 [7]. 

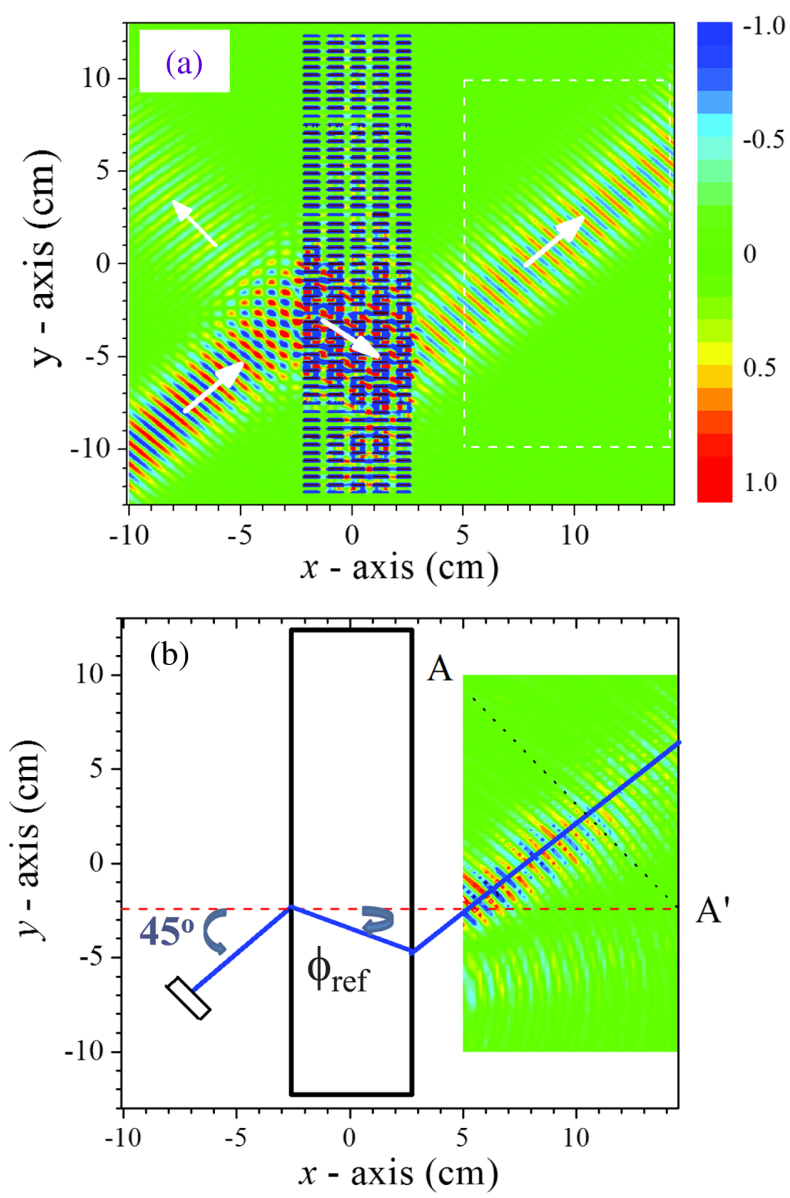

FIG. 3 (color online). (a) Calculated pressure map (real part) obtained from finite element simulations showing negative refraction. The cylindrical beam with frequency $40 \mathrm{kHz}$ is transmitted through five layers of holey Plexiglas plates (see Fig. 1). The white arrows indicate the directions of the beam. (b) Experimental data showing the pressure map measured in the rectangular area enclosed by dashed lines in part (a). The continuous lines are guides for the eye representing the directions followed by the beam. The horizontal dashed line defines the normal to the sample passing through the point contact of the impinging beam. The dotted line $A A^{\prime}$ is perpendicular to the direction of the transmitted beam.

Experimental measurements confirm the theoretical predictions. Firstly, negative refraction was demonstrated using a sample made of assembling five $12 \times 26 \mathrm{~cm}^{2}$ Plexiglas plates. These plates were milled and drilled with the geometrical parameters determined by the analytical model. Photographs of the sample are given in Fig. 1(a)-(b) and the caption reports its parameter dimensions. A scheme of the experimental setup employed in the measurements is described in Fig. 1(c). In brief, the sample is mounted in a rotary holder so that the angle of the incident beam with respect to the normal $\phi_{\text {inc }}$ can be manually selected. The incident beam travels a distance $d=7 \mathrm{~cm}$ from the emittertransducer (of diameter $25 \mathrm{~mm}$ ) to the front face of the sample. The emitted signal consists of a short chirp whose energy is distributed between 36 and $44 \mathrm{kHz}$. At the back side of the sample, a receiver-transducer (of diameter $8 \mathrm{~mm}$ ) is moved to scan the pressure over a rectangular area of $10 \times 20 \mathrm{~cm}^{2}$ with steps of $2.5 \mathrm{~mm}$, the longer side of this rectangle being parallel to the plates of the sample. At each position the receiver acquires the transmitted field, which is then processed through a Fourier transformations. Note that the emitted signal is pulsed and the reception is performed for a limited time to avoid unwanted reflections of the surrounding environment. The refractive angle $\phi_{\text {ref }}$ is determined through a geometrical procedure derived from the scheme shown in Fig. 1(c). Here the dotted line defined by the points $A$ and $A^{\prime}$ is perpendicular to the transmitted beam, which has the same angle as the incident one. From the point along this line where the pressure takes a maximum it is possible to know the position of the beam, and therefore the angle $\phi_{\text {ref }}$.

Figure 3(b) shows a snapshot of the measured pressure map for the angle $\phi_{\text {inc }}=45^{\circ}$ where negative refraction is clearly observed. Note that the additional beam appearing in the bottom of the scanned area is an artifact of our experimental setup produced by a side lobe of the emittertransducer. The behavior of this beam has been completely discarded in our discussions. A motion picture showing the pressure map as a function of the frequency is presented in the Supplemental Material Movie S2 [7].

The negative refraction behavior as a function of the frequency is clearly appearing in Fig. 4 which represents the pressure amplitude profiles taken along the line $A A^{\prime}$ depicted in Fig. 4(b). The white line joins the points with maximum amplitude and, therefore, represents the frequency dependence of $\phi_{\text {ref }}$ for the given incident angle, $\phi_{\text {inc }}=45^{\circ}$. The simulations using the finite element method [see Fig. 4(a)] overestimate the refractive angles obtained from the measurements [see Fig. 4(b)], but both give support to our claim of negative refraction associate to these structures for a broad span of angles and frequencies. The complexity of the threedimensional simulations which involve a huge number of finite elements and the differences between the actual exciting beam with the Gaussian beam employed in the numerical algorithm are the reasons explaining the discrepancies observed between theory and experiments. Note that the Gaussian beam is two dimensional, with the same values along the vertical $z$ axis, while the sound excited by the emitter transducer is a true three-dimensional beam.

Negative refraction was previously demonstrated using acoustic metamaterials based on space coiling [8,9], the drawbacks of these structures being their difficult fabrication in comparison with those studied here and their strong lossy characteristics [10]. Negative refraction was also demonstrated in phononic crystals with a fluid (liquid or air) matrix surrounding solid inclusions $[11,12]$ and recently using a foamlike metallic structure [13]. All of them are based on the negative slope of some high frequency bands in the dispersion relation. Our demonstration, which is based 

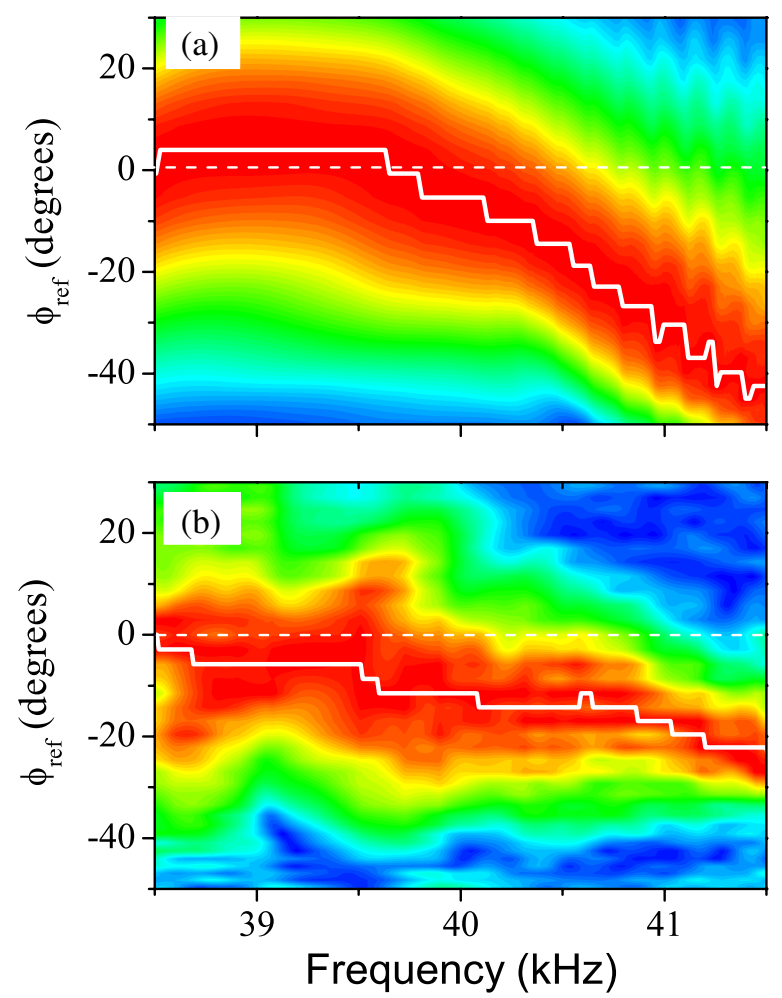

FIG. 4 (color online). Frequency dependence of the normalized pressure amplitude taken along the line $A A^{\prime}$ represented in Fig. 1(c). (a) Calculated propagating angle, $\phi_{\text {ref }}$, inside the hyperbolic material. (b) Measured angle. Both results are obtained considering that the direction of the incident beam is fixed at $\phi_{\mathrm{inc}}=45^{\circ}$, as in Fig. 3. The white curves follows the level with maximum amplitude. The horizontal lines define the zero.

on strongly hyperbolic dispersion, can be considered as a structure with less fabrication demands and difficulties. Furthermore it does support further functionalities as compared to other configurations. Another advantage is that the structure is tunable; i.e., the parameters are scalable to work at a given prefixed frequency.

Energy funneling is obtained with strongly anisotropic hyperbolic materials; i.e., when the bottom of the hyperboloid is extremely flat as is the case in the structures under study here (see the inset of Fig. 2). Thus, the waves arriving to the material surface within certain incident angles are refracted into a direction normal to the surface. The resulting collimated beam experiences a ballistic transmission inside the material bulk until the sample rear side where it is radiated to the free space. Eventually an acoustic waveguide with the same thickness than the beam could be added to insert the outgoing energy into an acoustic circuit.

The experimental demonstration of the funneling effect has been performed here using a sample constructed by assembling nine Plexiglas plates, the emitter-transducer being placed at $d=7 \mathrm{~cm}$ from the sample front surface. According to the scheme in Fig. 1(c), the sound pressure was acquired at position $P_{1}$ as a function of the incident angle $\phi_{\text {inc }}$, in steps of $2.5^{\circ}$. Figure 5 shows the frequency

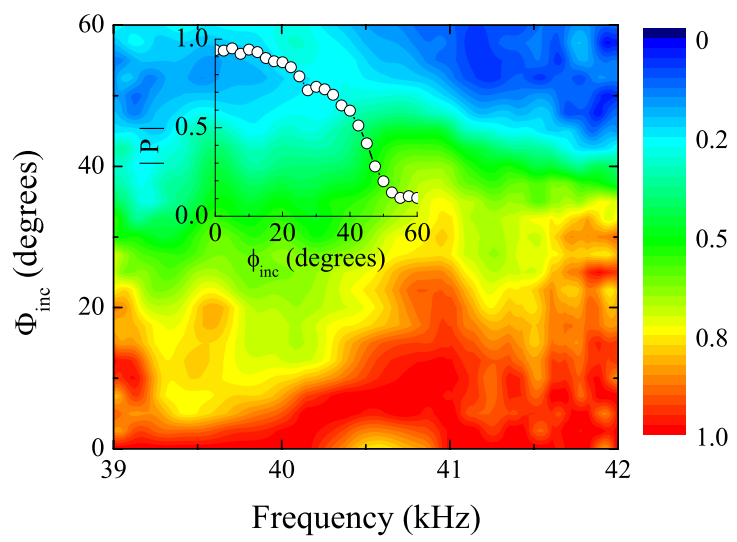

FIG. 5 (color online). Experimental data showing the frequency dependence of the pressure amplitude measured at $P_{1}$ as a function of the incident direction [see Fig. 1(c)]. The inset depicts the profile taken at $41 \mathrm{kHz}$. Experiments have been performed using a sample made of nine holey plates.

dependence of the measured pressure amplitude for incident angles between $0^{\circ}$ and $60^{\circ}$. The main feature characterizing the funneling effect is clearly observed; that is, the pressure at $P_{1}$ does not change against strong variations of the incident angle. This feature is particularly remarkable for frequencies between 40.5 and $42 \mathrm{kHz}$. The inset shows the profile taken at $41 \mathrm{kHz}$, where the pressure profile is practically flat for angles between $0^{\circ}$ and $22^{\circ}$, confirming the predictions of the calculated hyperbolic profile depicted in Fig. 2. A motion picture showing the frequency dependence of the emitted beam propagating in free space is presented in the Supplemental Material Movie S3 [7].

This is not the first demonstration of funneling of acoustic energy with perforated structures, but the previous one $[14,15]$ employed a metallic layer with a single hole

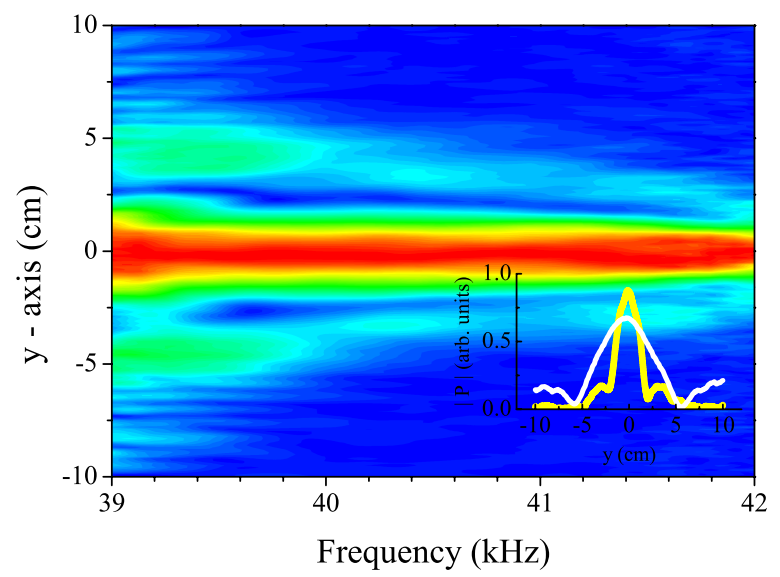

FIG. 6 (color online). Pressure profile of the beam transmitted through a sample made of nine holey plates. The frequency dependence of the beam profile is measured along the $y$ axis separated $2 \mathrm{~cm}$ from the back surface. The inset shows the profiles taken at $41 \mathrm{kHz}$ with (yellow line) and without (white line) the sample. 
and the phenomenon was obtained resonantly, using acoustic surface waves. Here, on the contrary, it is a nonresonant phenomenon and consequently its bandwidth is not strongly limited.

Another interesting phenomenon that is also related to the hyperbolic band profile is the squeezing and collimation of the emitted beam. Both effects squeezing and collimation are consequences of the strong anisotropy of the hyperbolic EFS: for the larger incident angles the beam is negatively refracted while for all the angles smaller than a certain value, the beam is collimated along the direction normal to the surface. The experimental data demonstrating these effects are reported in Fig. 6. The frequency dependence of the beam measured after passing through the sample is given in the Supplemental Material Movie S4 [7].

In summary, we have demonstrated negative refraction and energy funneling of airborne sound by simple artificial structures made of assembling a few holey Plexiglas plates. It is shown that these structures show hyperbolic dispersion bands, a feature providing ingenious ways to control and manipulate sound waves, equivalent to those proposed in optics and microwaves [1,2]. Acoustic hyperlenses and spot-size converters will allow the application of the phenomena reported here for acoustic imaging and acoustic circuits, respectively.

This work was partially supported by the Office of Naval Research (USA) under Grant No. N000140910554, and by the Ministerio de Economía y Competitividad (Spain) under Contract No. TEC2010-19751. J.C. gratefully acknowledges financial support from the Danish Council for Independent Research and a Sapere Aude Grant (12-134776).
*Corresponding author.jsdehesa@upv.es

[1] A. Poddubny, I. Iorsh, P. Belov, and Y. Kivshar, Nat. Photonics 7, 948 (2013).

[2] A. V. Kildishev, A. Boltasseva, and V. Shalaev, Science 339, 1232009 (2013).

[3] J. Li, L. Fok, X. Yin, G. Bartal, and X. Zhang, Nat. Mater. 8, 931 (2009).

[4] D. Torrent and J. Sánchez-Dehesa, New J. Phys. 13, 093018 (2011).

[5] J. Christensen and F. J. Garcia de Abajo, Phys. Rev. Lett. 108, 124301 (2012).

[6] J. Christensen and F. J. Garcia de Abajo, Phys. Rev. B 86, 024301 (2012).

[7] See Supplemental Material at http://link.aps.org/ supplemental/10.1103/PhysRevLett.112.144301, where details are given of the numerical simulations.

[8] Z. Liang and J. Li, Phys. Rev. Lett. 108, 114301 (2012).

[9] Y. Xie, B.-I. Popa, L. Zigoneanu, and S. A. Cummer, Phys. Rev. Lett. 110, 175501 (2013).

[10] T. Frenzel, J. D. Brehm, T. Buckmann, R. Schittny, M. Kadic, and M. Wegener, Appl. Phys. Lett. 103, 061907 (2013).

[11] S. X. Yang, J. H. Page, Z. Y. Liu, M. L. Cowan, C. T. Chan, and P. Sheng, Phys. Rev. Lett. 93, 024301 (2004).

[12] A. Sukhovich, L. Jing, and J. H. Page, Phys. Rev. B 77, 014301 (2008).

[13] A.-C. Hladky-Hennion, J. O. Vasseur, G. Haw, C. Croenne, L. Haumesser, and A. Norris, Appl. Phys. Lett. 102, 144103 (2013).

[14] J. Christensen, A. Fernandez, F. de Leon Pérez, L. MartínMoreno, and F. J. García-Vidal, Nat. Phys. 3, 851 (2007).

[15] Y. Zhou, M.-H. Lu, L. Feng, X. Ni, Y.-F. Chen, Y.-Y. Zhu, S.-N. Zhu, and N.-B. Ming, Phys. Rev. Lett. 104, 164301 (2010). 